\title{
Artificial Intelligence in Neuroanesthesiology and Neurocritical Care
}

\author{
Vanitha Rajagopalan ${ }^{1, \odot}$ Dilip K. Kulkarni² \\ ${ }^{1}$ Department of Neuroanaesthesiology \& Critical Care, All India \\ Institute of Medical Sciences (AlIMS), Ansari Nagar, New Delhi, \\ India \\ 2Department of Anaesthesia, Malla Reddy Narayana Multispeciality \\ Hospital \& Malla Reddy Medical College for Women, Suraram, \\ Hyderabad, Telangana, India \\ J Neuroanaesthesiol Crit Care 2020;7:11-18
}

\begin{abstract}
Address for correspondence Dilip K. Kulkarni, MD, Department of Anaesthesia, Malla Reddy Narayana Multispeciality Hospital \& Malla Reddy Medical College for Women, Suraram, Hyderabad 500055, Telangana, India (e-mail: dilipkum@gmail.com).
\end{abstract}

\begin{abstract}
Keywords

- artificial intelligence

- machine learning

- neuroanesthesiology

- neurocritical care

Artificial intelligence (AI) already influences almost every sector of our daily life, including the rapidly evolving technologies and datasets of healthcare delivery. The applications in medicine have significantly evolved over the past few decades and have shown promising results. Despite constant efforts to incorporate $\mathrm{Al}$ into the field of anesthesiology since its inception, it is still not commonplace. Neuroanesthesiology and neurocritical care is a discipline of medicine that deals with patients having disorders of the nervous system comprising a complex combination of both medical and surgical disease conditions. Al can be used for better monitoring, treatment, and outcome prediction, thereby reducing healthcare costs, minimizing delays in patient management, and avoiding medical errors. In this review, we have discussed the applications of $\mathrm{Al}$ and its potential in aiding the clinician's judgment in several aspects of neuroanesthesiology and neurocritical care, some of the barriers to its implementation, and the future trends in improving education in this field, all of which will require further work to understand its exact scope.
\end{abstract}

\section{Introduction}

The official introduction of AI occurred during the 1956 Dartmouth Artificial Intelligence Conference. John McCarthy coined the term "artificial intelligence" in 1955, and is one of the "founding fathers" of artificial intelligence together with Alan Turing, Marvin Minsky, Allen Newell, and Herbert A. Simon. ${ }^{1}$ Since then, there has been widespread implementation of $\mathrm{AI}$ in all sectors including healthcare ${ }^{2,3} \mathrm{AI}$ is defined as the development of computer systems to model intelligent behavior with minimal human intervention.

The application of AI in medicine has two main branches: 1) virtual branch and 2) physical branch.

The physical branch comprises highly repetitive work. It empowers the doctors to deliver faster and more accurate clinical care by offering them expertise and assistance. The virtual component is represented by Machine Learning (ML), mathematical algorithms that improve learning through experience.

\section{Health Data Management}

The health data are nowadays available in electronic health records (EHRs). The medical data include numerical information, laboratory test results, genetic tests, culture results, images, treatment information, administrative data, and health research information. Clinical data stored in EHR are both structured and unstructured ${ }^{4,5}$ ( - Fig. 1).

\section{Structured Data}

Structured data follow a prescribed data model and value set, constraining the users to only be able to choose
DOI https://doi.org/ 10.1055/s-0040-1701954 ISSN 2348-0548.
License terms

()(1) $\odot \circledast$ 


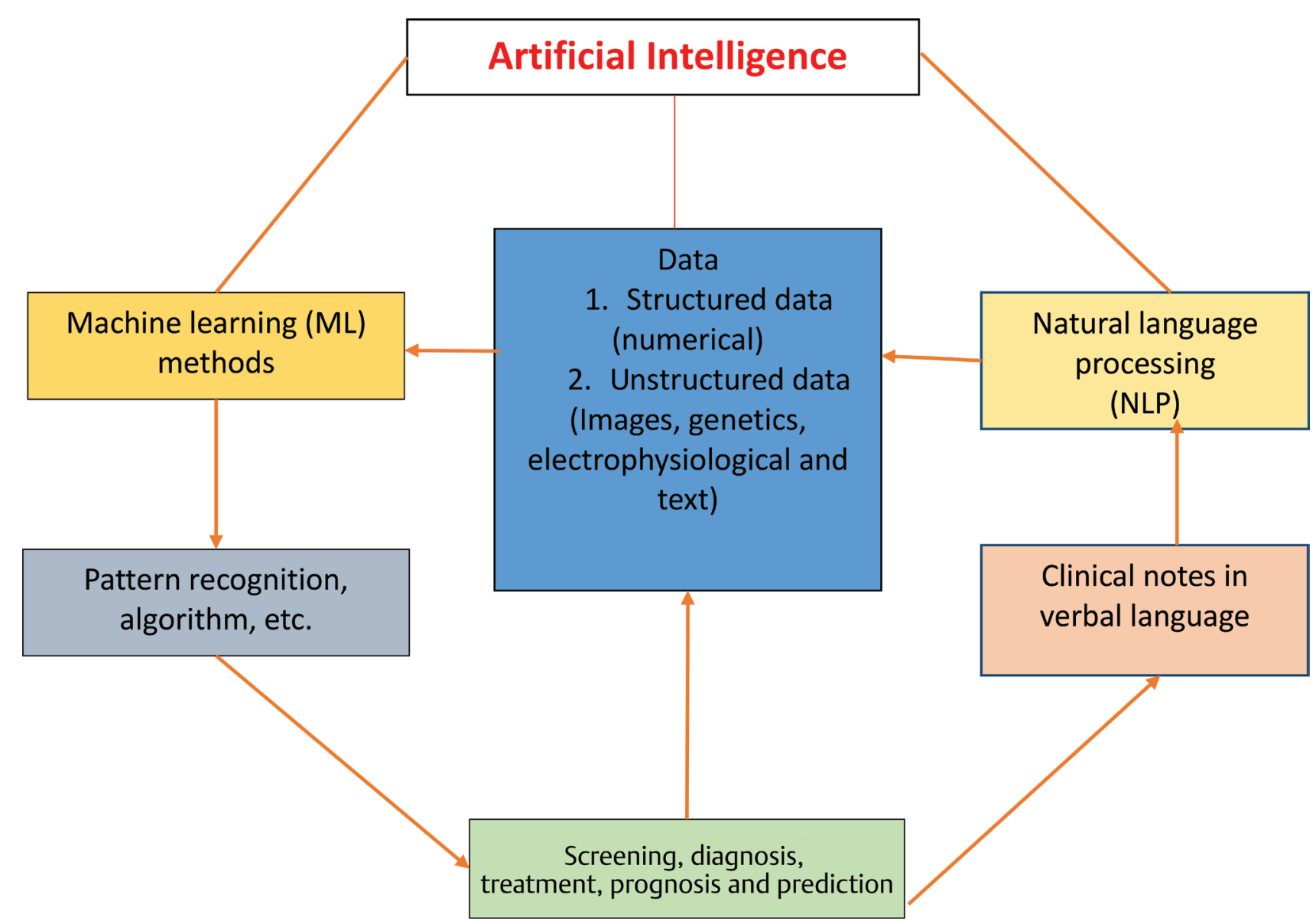

Fig. 1 The cycle of medical data generation through medical health records, NPL to ML to clinical treatment and prediction. ML, machine learning; NPL, natural language processing.

predetermined values. Computers can readily process structured data. Data sent by medical devices to EHRs are usually structured data.

\section{Unstructured Data}

Unstructured data do not follow a predefined set of values, allowing users to instead enter narrative information about data using their own words. This means recording data provides the user with the most freedom for recording an entry, but because the same clinical event could be documented in myriad ways, computers cannot easily process unstructured data, making errors more likely. These data have to be converted to computer readable data through the natural language processing (NLP) methods. ${ }^{5,6}$

\section{Machine Learning Algorithms}

Machine learning (ML) can be classified as follows, ${ }^{4,-9}$ :

1. Unsupervised

2. Supervised

3. Deep learning

4. Reinforcement learning

\section{Unsupervised Machine Learning}

Unsupervised learning is a ML technique, where the model works on its own to discover information and the outcomes of the model are not defined.

It performs more complex processing tasks compared with supervised learning but becomes unpredictable compared with other ML techniques and is less accurate.

Clustering, association, and principal component analysis (PCA) fall into unsupervised techniques. Clustering finds out the structure and pattern in data and identifies different groups, whereas association establishes the relationship in the datasets from the given database. PCA is mainly used for the dimension reduction of data.

\section{Supervised Machine Learning}

The input and output variables are provided in a supervised learning model. Thus, specified data are used to train algorithms, and a link is established between input and output variables in a supervised learning model. These techniques are highly precise.

The supervised learning techniques are regression and classification. Classification separates the data, whereas regression fits the data. 


\section{SVM Polynomial Decision Boundary}

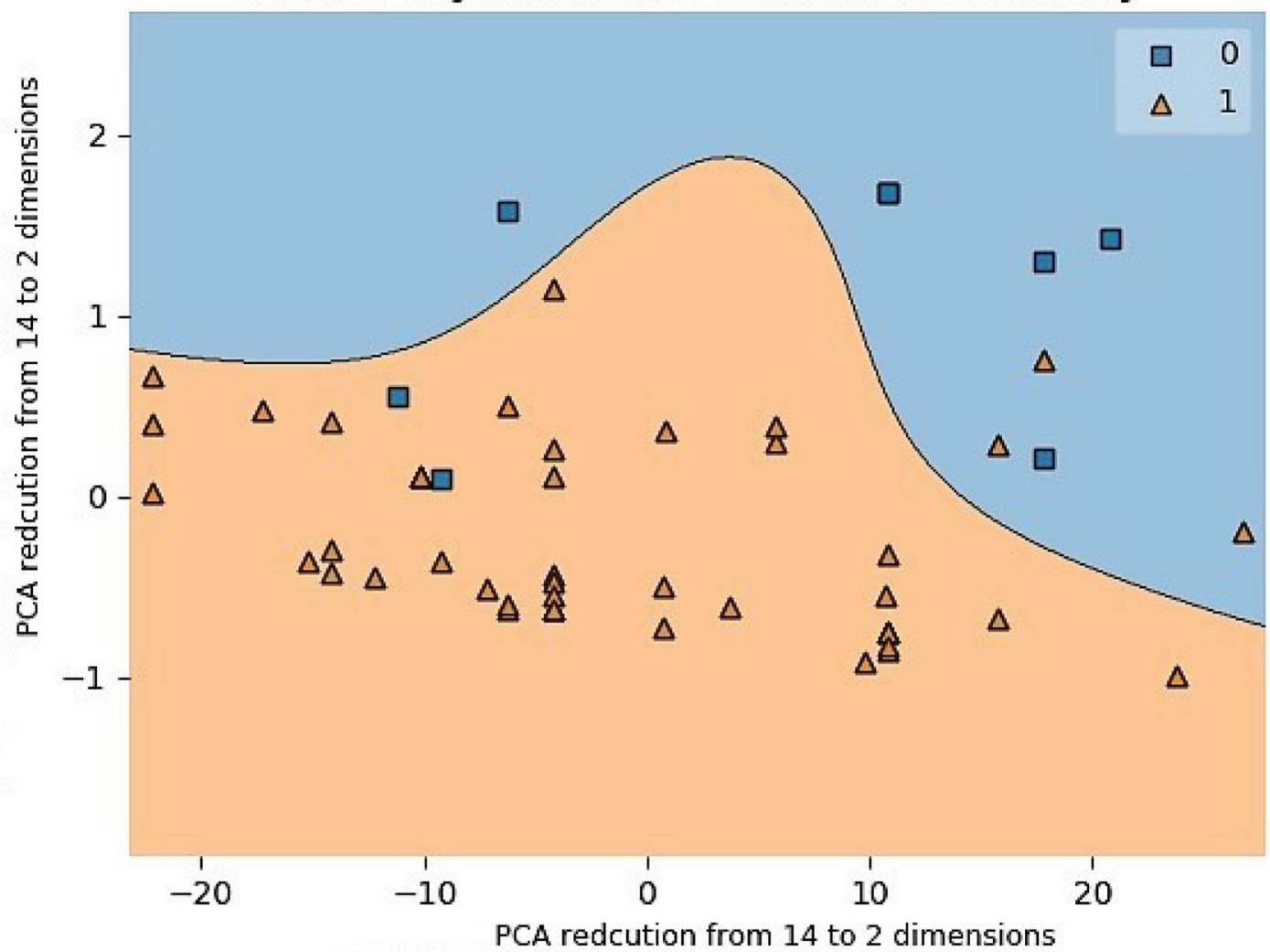

Fig. 2 Depiction of SVM classifying the data into two categories and data reduction by PCA. PCA, principal component analysis; SVM, supervised learning algorithm.

The following algorithms are used in supervised ML techniques: decision tree, random forest (RF), Naïve Bayes (NB), support vector machine (SVM), artificial neural networks (ANN), discriminant analysis, nearest neighbor, linear regression, and logistic regression. The SVM and ANN are frequently used in the medical field.

\section{Support Vector Machine}

This supervised learning algorithm classifies the data into two categories. The model is built from the data already sorted in two categories ( - Fig. 2). This makes SVM a kind of nonbinary linear classifier. SVMs are used in text categorization, image classification, prediction, and handwriting recognition.

\section{Artificial Neural Network}

The neural network captures complex nonlinear relationships between input and outcome variables by multiple hidden layers (HLs) with prior specified functions. The weights are established through the input and outcome data; thus, the average error is reduced and the predictions become more accurate ( - Fig. $\mathbf{3}$ ).

\section{Deep Learning}

Deep learning is a self-teaching system in which the existing data are used to train algorithms to find the patterns and then make predictions about new data. The ANNs with multiple layers of nodes create deep learning algorithms that mimic the network of neurons of the brain. This algorithm with multiple cycles defines patterns and improves the precision of predictions with each cycle ( $\boldsymbol{- \text { Fig. }} \mathbf{4}$ ).

\section{Reinforcement Learning}

Like deep learning, reinforcement learning is autonomous. But deep learning is learning from a training set and then applying that learning to a new dataset, while reinforcement learning is dynamically learning by adjusting actions, based on continuous feedback, to maximize a reward. ${ }^{3,6-8}$

Neuroanesthesiology and neurocritical care as a discipline is rendered difficult due to the inherent limitations in the assessment of patients with neurological injury. As neuroanesthesiologists, we work in operating rooms and intensive care units (ICUs), both being acute care settings, which demand vigilance, steady hands, and quick thinking.

AI can definitely assist us in making better clinical decisions and provide up-to-date medical information from journals, textbooks, and clinical practices. This results in early diagnosis, predicts outcome of the disease as well as treatment, provides feedback on treatment, and reduces errors. This greatly increases patient safety and saves costs. These traits allow AI systems to continuously monitor and treat neurocritical care patients in real-time. Early signs of 


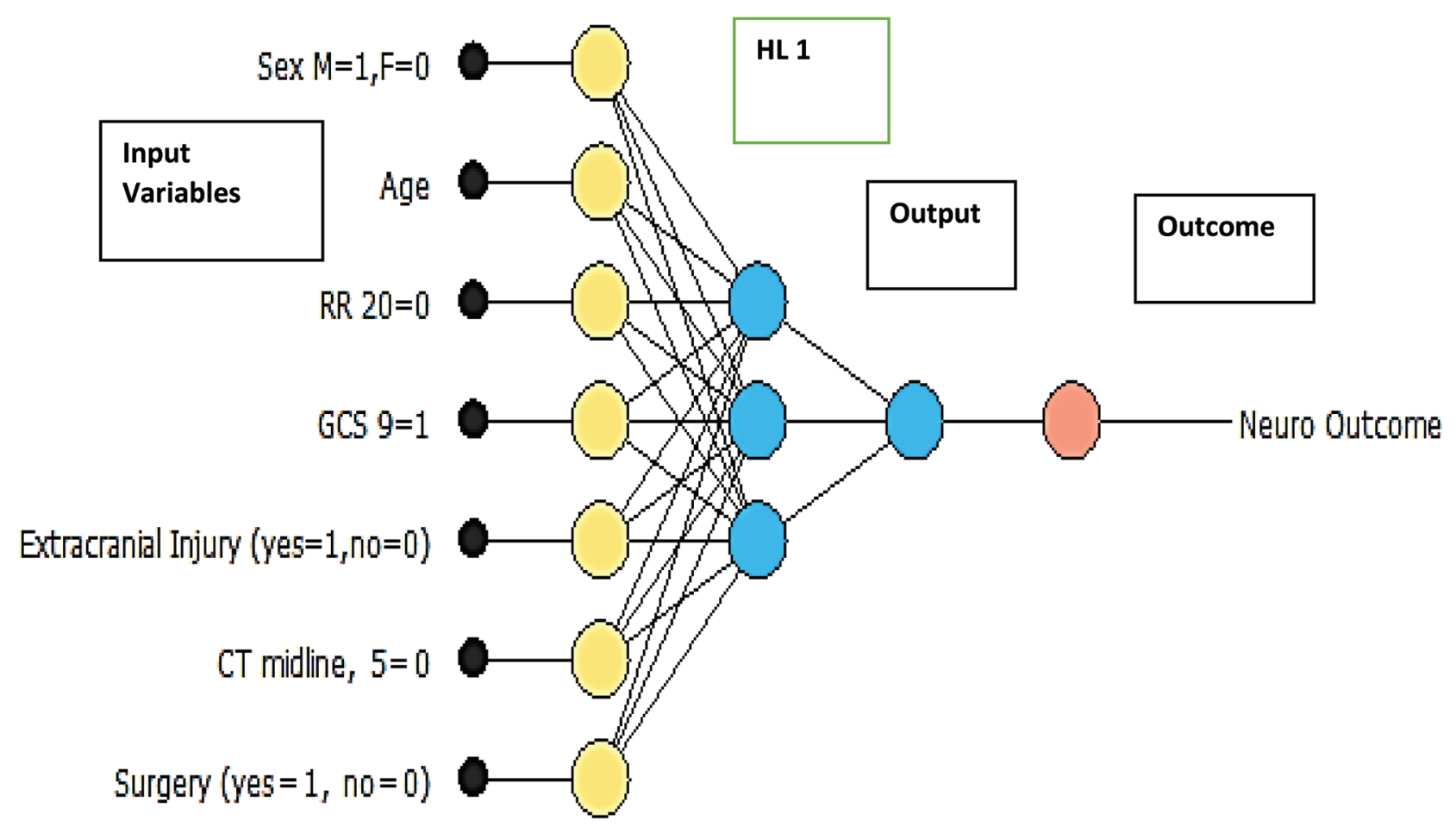

Fig. 3 A diagram representing ANN, showing the outcome of head injury; the input variables are sex (M-male, F-female), age, RR, GCS, extracranial injuries, CT scan of the midline shift, and whether surgery was performed in binary fashion. The HL is only one. ANN, artificial neural network; CT, computed tomography; GCS, Glasgow coma scale; HL, hidden layer; RR, respiratory rate.

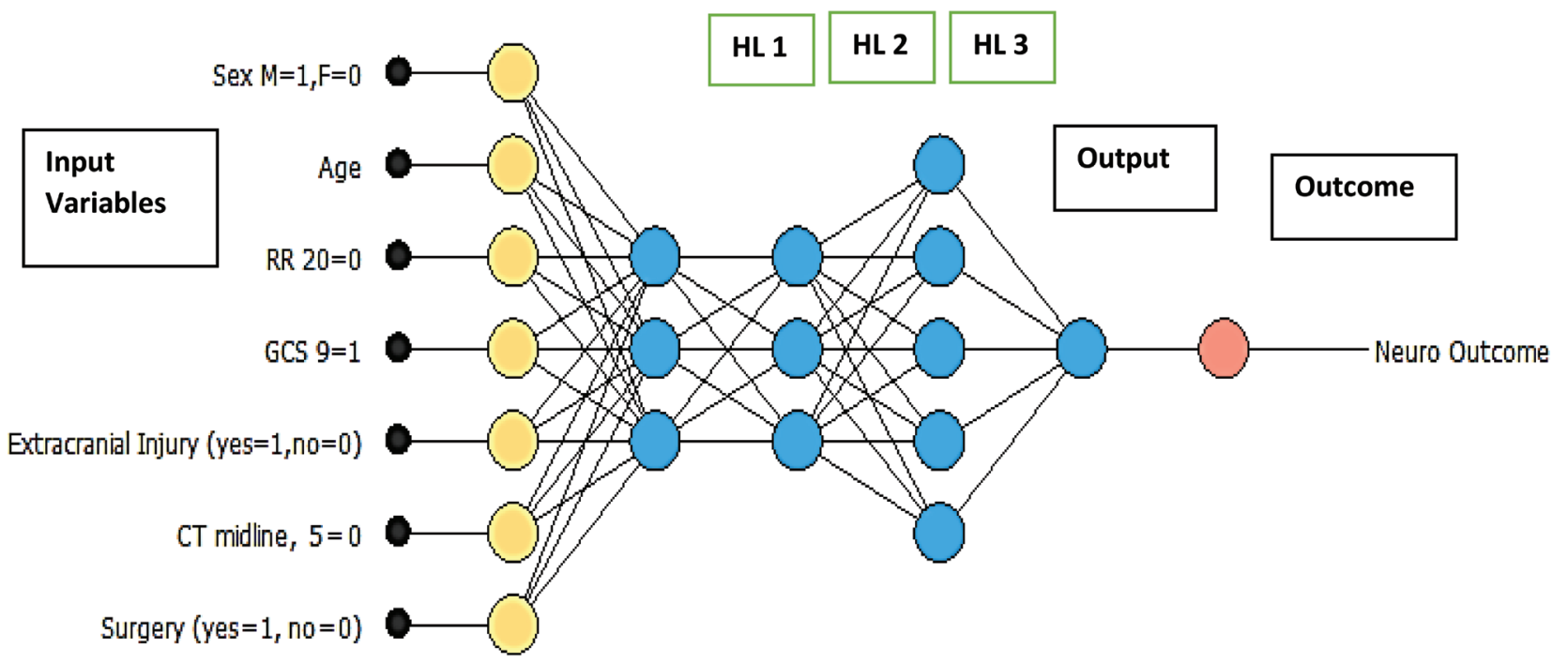

Fig. 4 Depiction of deep learning of ANN with the following input variables: sex (M-male, F-female), age, RR, GCS, extracranial injuries, CT scan of the midline shift, and whether surgery was performed in the binary fashion with three hidden layers of nodes and the head injury outcome. ANN, artificial neural network; CT, computed tomography; GCS, Glasgow coma scale; HL, hidden layer; RR, respiratory rate.

neurological deterioration could be detected more promptly and appropriate measures taken more quickly, thereby improving patient outcomes. AI can also help patients in areas where neurocritical care is not available, ${ }^{10}$ as they take over more of the basic patient management, by analyzing the data and titrating treatments in real-time, reducing possible delays in patient care and optimizing the patient's condition till he/she is transferred to a higher center with neurocritical care facility.

In this review, we have discussed the applications of $\mathrm{AI}$ in neuroanesthesiology and neurocritical care, the barriers to its implementation, and the future trends in this field. 


\section{Applications in Neuroanesthesiology and Neurocritical Care}

AI creates a potential system to manage the neuroanesthesiology and neurocritical care patient with minimal or no supervision, freeing the clinician to focus attention elsewhere. ${ }^{11-13}$ Some potential parameters include anesthetics/analgesics, antiepileptic drugs (AEDs), blood pressure, glucose, fluids/ electrolytes, neuromuscular blockade, and ventilator settings. ${ }^{14-25}$ The applications of AI in our field can be broadly discussed under five categories (1) predictive analytics, (2) imaging, (3) smart devices, (4) Administration, and (5) research and education.

\section{Predictive Analytics}

Much of the work in critical care using AI has focused on predictive analytics. Improvement in the prediction of adverse events such as hypotension has been shown using advanced ML models in critical care environments. ${ }^{26} \mathrm{ML}$ methods can predict the risk of postinduction hypotension. ${ }^{27,28}$ These models enables detection and intervention up to 15 minutes before an event and have been generalized for use in the multicenter clinical environment. ${ }^{29}$ Predictive therapeutic interventions to prevent hypotension using $\mathrm{AI}$ have also been constructed for fluid resuscitation. ${ }^{30}$ By combining models for early hypotension detection and therapeutic intervention, there is potential to prevent or minimize patient deterioration and the subsequent development of multisystem organ dysfunction.

Sepsis remains one of the largest causes of mortality in the ICU. Sepsis algorithms become more important and these interpretable models can predict sepsis 4 to 12 hours before clinical recognition. ${ }^{31}$

ML models can also help in the prediction of hospital-acquired infections such as central line-associated blood stream infection and Clostridium difficile infections. ${ }^{32}$

Prediction of prolonged mechanical ventilation is useful for early tracheostomy, ventilator weaning, and rehabilitation. Use of AI to identify patients who will require $>7$ days of mechanical ventilation has been shown to improve outcomes. ${ }^{33}$ Teams are also using AI to aid ventilator weaning by targeting the success of extubation. Kuo et a ${ }^{34}$ used neural networks to create a model with an accuracy of $80 \%$ and improved on traditional prediction by rapid shallow breathing index.

AI has also been implemented in specialized ICUs such as in neurointensive care for early and accurate risk assessment of seizures in critically ill patients. ${ }^{35}$

Predictive ML models for patient trajectories ${ }^{36}$ and ICU readmission have been developed and have shown higher predictive values than the conventionally used stability and workload index for transfer score or the modified early warning score criteria for early deterioration. ${ }^{37}$

Mortality is a common outcome in medical studies, and prediction capabilities related to it have been studied extensively using ML and NLP. Use of NLP enables inclusion of the traditionally difficult-to-use clinical notes. Weissman et $a^{38}$ showed the ability to use unstructured data such as clinical notes, and terms such as "poor prognosis," by using various NLP techniques. Using neural networks, classification algorithms can be constructed for identification of the most important terms in physician notes, which then can be used to construct ML models to predict outcomes such as mortality in the surgical ICU..$^{39}$ One such model, called Early Mortality Prediction for Intensive Care Unit patients, has been shown to outperform traditional scoring systems such as acute physiology and chronic health evaluation (APACHE) and sequential organ failure assessment (SOFA) despite missing values within the training datasets. The area under the curve (AUC) is $0.82 \pm 0.04$ compared with the traditional scoring systems which range from 0.54 to $0.65 . .^{40}$ This model has not only focused on prediction accuracy, but has also attempted to generate earlier prediction by hours using multimodal data. The benefits of such models once again lie in triage, early intervention, and appropriate treatment recommendation to minimize risk to the patient and provide for cost-effective care.

Harnessing meaningful information from EHR data and data registries is expensive, can be of limited value, and is utilized primarily for retrospective research analysis. As we have seen from the above examples, ML provides a more cost-effective way to carry out retrospective research and, in constructing models, can provide real-time or prospective guidance to clinicians. ${ }^{41}$

Machine-learning models have been developed for predicting mortality following trauma in motorcycle riders. ANNs have been used to predict outcome following head injury. ${ }^{42,43}$

The occurrence of symptomatic cerebral vasospasm (SCV) after aneurysmal subarachnoid hemorrhage (aSAH) is a morbid and common problem. A simple ANN model was found to be more sensitive and specific than multiple logistic regression (MLR) models in prediction of SCV in patients with aSAH ${ }^{44} \mathrm{ML}$ has been used to predict outcome in intracranial aneurysms treated with flow diverters. ${ }^{45}$

Hollon et al ${ }^{46}$ sought to build a predictive model using supervised ML to accurately predict early outcomes of pituitary adenoma surgery. These results provide insight into how predictive modeling using ML can be used to improve the perioperative management of pituitary adenoma patients.

Stroke is one of the major causes of disability and death worldwide. It is estimated that up to $80 \%$ of strokes can be prevented if one can identify or predict the occurrence of stroke in its early stage. ${ }^{47}$ AI-based methods offer several advantages in improving prediction performance for stroke treatment, prognosis, and functional outcome prediction. This helps neurophysicians to identify high-risk patients and guide treatment approaches, leading to decreased morbidity. Several AI-based techniques are being investigated to develop automated platforms for precisely predicting prognosis and the functional outcome. Park et a ${ }^{48}$ have proposed a Bayesian network model for the prediction of poststroke outcomes with the available risk factors. They also introduced an online "Yonsei stroke outcome inference system" for predicting functional independence at 3 months and mortality within 1 year in patients with stroke using the Bayesian network model. 
The timely diagnosis of stroke is crucial for good functional recovery and minimizing mortality. AI offers technology solutions with high-precision and accuracy for the diagnosis of stroke, its severity, as well as prediction of functional outcomes. ${ }^{49}$

Recently, in diagnostic neuroradiology, there has been an interest in adopting $\mathrm{AI}$ and $\mathrm{ML}$ techniques ${ }^{50,51}$ and in the prediction of the outcome in patients postneurointerventional procedures. ${ }^{52,53}$ Two recent studies ${ }^{52,53}$ have used ANN modeling and supported vector machine algorithms in prediction of the final Modified Rankin Score (mRS) with relatively good accuracy and precision. The accuracy of outcome prediction, using supervised ML algorithms has shown promising results, especially in the prediction of final outcome as good or bad as well as the probability of requiring retreatment in future, with the potential for incorporation of larger multicenter datasets, which will further improve predictive accuracy. $^{54}$

\section{Imaging}

Point-of-care ultrasound for assessment of cardiac function, volume status, and vasopressor/inotrope management has witnessed increasing utilization in care of critically ill patients. Deep learning models have been developed that can enable fast and accurate classification of cardiac anatomy on echocardiograms..$^{55}$ Innovations such as these are likely to propel clinicians into a newer era of enhanced integration of various imaging techniques to generate more accurate diagnosis and treatment methods. Automated analysis of medical imaging is a prominent area in ML applications. ML models have been implemented in the reading of radiographic images, including X-rays and computed tomographic scans, and have reported increasing accuracy for clinical diagnosis. ${ }^{56}$

Within the ICU, models have been developed to provide surveillance for lines and tubes to assess proper device positioning. ${ }^{57}$ In addition, waveform analysis from ventilator data has been used to create models to detect patient-ventilator asynchrony that match clinical experts. ${ }^{58}$ Thus far, the primary use of ML waveform analysis has been to either automatically screen waveforms such as electrocardiograms and electroencephalographs, which cannot be monitored constantly by clinicians. The goal of these models is to improve time to early intervention. ${ }^{59,60}$ The tools developed for waveform detection could also be used to reduce the burden of alarms plaguing ICUs. One of the Joint Commission International goals is to reduce alarm fatigue among care providers, which can conceivably be achieved using modern AI techniques. ${ }^{61}$

\section{Smart Devices}

Medication delivery and titration is a key component of patient care in the ICU and requires a large amount of clinical resources. Smart pumps exist for medication titration, and these devices can be further utilized for their abilities to provide closed loop management. In future, increased utilization of closed loop infusions will hopefully decrease manual labor while possibly enhancing consistency in steady-state drug delivery. Models using unsupervised learning have been trialed for clinical applications, including use in vasopressor drug delivery in the ICU.62 Similarly, for tight glycemic control, AI-based artificial pancreas systems have been developed for use in the ICU. ${ }^{63}$

\section{Administration}

Triage from emergency departments is a complicated task and includes identification of high-risk patients who need to be promptly admitted to the ICU. AI models have been developed that can help triage trauma patients, thereby leading to appropriate and timely resource utilization. ${ }^{64,65}$ Similarly, identification of cohorts of patients with similar clinical needs has been postulated to provide a framework for future organizational innovations in the ICU and provide better cost-effective care. ${ }^{66}$

\section{Research and Education}

Considerable research has been generated in all areas of AI. AI in medical education is still in its infancy. In the future, it is likely that basic understanding of AI and its applications will be required in clinical practice and thus will be part of educational curricula to facilitate better understanding, interpretation, and implementation.

Anesthesiologists and intensivists work at the junction of many disciplines: surgery, medicine, biology, pharmacology, mathematics, and physics, and are well-placed to embrace modeling. They have access to knowledge and expertise of enormous breadth and have experience of a huge array of induced and pathological states, and are comfortable with biological science, physical science, numbers, technology, and medicine. Anesthesiologists and intensivists, above all, have clinical contact, a real understanding of real-world relevancy and empathy for the issues of importance. In addition, they have skills in managing teams of individuals, collaborating and coordinating their efforts toward a single goal. All that is required of the researcher who wants to use modeling is to get an idea of what may be achieved and find a suitable question to answer. Contact with an expert will be enormously helpful during the researcher's early forays into AI.

\section{Limitations and Future Trends}

AI technologies have great potential for assisting future neuroanesthesiologists and neurocritical care physicians. However, significant hurdles remain before they can be used routinely in the operating room and ICU. One of the most significant challenges is creating adequate regulatory mechanisms to ensure the patients under the care of AI are safe and protected. When an $\mathrm{AI}$ independently decides to intervene on a patient and causes an adverse event, who is then held accountable? Furthermore, patient autonomy might be challenged because the AI system can administer care in an automated fashion without involving the patient in the decision-making process. The patient-doctor relationship can become more "distant," as these AI systems take over more of the basic patient management. As this technology becomes more widespread, these challenges need to be addressed before AI can play any role in patient care. 


\section{Conclusions}

As a specialty, neuroanesthesia and critical care should continue to create and refine real-time evidence-based, individualized, clinical decision supportive tools and guidelines. One of the future clinician's greatest challenges will be validating the safety and efficacy of these systems. The revolution in $\mathrm{AI}$ is so much that there may be a risk of technological collusions such as cloud and edge computing to even surpass human intelligence in the years to come. The challenge for healthcare professionals, in particular, is the willingness to accept technology and the transformation that is inevitable. Effective clinical governance to ensure patient safety is vital for digital health.

\section{Conflict of Interest}

None declared.

\section{Acknowledgments}

We would like to thank Mr. Akshay Bhavani Kumar Kulkarni, software engineer working at Teradata, Hyderabad, for constructing the machine learning algorithm illustrations.

\section{References}

1 McCarthy J, Minsky ML, Rochester N, Shannon CE. A proposal for the dartmouth summer research project on artificial intelligence. AI Mag 2006;27(4):12-14

2 Russell SJ, Norvig P. Artificial Intelligence: A Modern Approach Prentice Hall Series in Artificial Intelligence. 3rd ed. Upper Saddle River, NJ: Prentice Hall; 2010:xviii, 1132

3 Jordan MI, Mitchell TM. Machine learning: trends, perspectives, and prospects. Science 2015;349(6245):255-260

4 Jiang $\mathrm{F}$, Jiang $\mathrm{Y}$, Zhi $\mathrm{H}$, et al. Artificial intelligence in healthcare: past, present and future. Stroke Vasc Neurol 2017;2(4):230-243

5 Praveen S, Chandra U. Influence of structured, semi-structured, unstructured data on various data models. Int J Sci Eng Res 2017;8(12):67-69

6 Scholte M, van Dulmen SA, Neeleman-Van der Steen CWM, van der Wees PJ, Nijhuis-van der Sanden MW, Braspenning J. Data extraction from electronic health records (EHRs) for quality measurement of the physical therapy process: comparison between EHR data and survey data. BMC Med Inform Decis Mak 2016;16(1):141

7 Langley P, Carbonell JG. Approaches to machine learning. J Am Soc Inf Sci 1984;35:306-316

8 Osisanwo FY, Akinsola JET, Awodele O, et al. Supervised machine learning algorithms: classification and comparison. Int J Comput Trends Tech 2017;48(3):128-138

9 The professional experts in data science. Neural DesignerEditor. Villamayor, Salamanca (Spain): Artificial Intelligence Techniques, Ltd; 2019. Available at: www.neuraldesigner.com/

10 Hanson CW III, Marshall BE. Artificial intelligence applications in the intensive care unit. Crit Care Med 2001;29(2):427-435

11 Bennett CC, Hauser K. Artificial intelligence framework for simulating clinical decision-making: a Markov decision process approach. Artif Intell Med 2013;57(1):9-19

12 Uemura K, Sugimachi M. Automated cardiovascular drug infusion system to control hemodynamics. Adv Biomed Eng 2013;2:32-37

13 Rinehart J, Liu N, Alexander B, Cannesson M. Review article: closed-loop systems in anesthesia: is there a potential for closed-loop fluid management and hemodynamic optimization? Anesth Analg 2012;114(1):130-143

14 Tehrani FT. A closed-loop system for control of the fraction of inspired oxygen and the positive end-expiratory pressure in mechanical ventilation. Comput Biol Med 2012;42(11):1150-1156

15 Tehrani F, Rogers M, Lo T, et al. A dual closed-loop control system for mechanical ventilation. J Clin Monit Comput 2004;18(2):111-129

16 Wysocki M, Brunner JX. Closed-loop ventilation: an emerging standard of care? Crit Care Clin 2007;23(2):223-240, ix ix

17 Lellouche F, Brochard L. Advanced closed loops during mechanical ventilation (PAV, NAVA, ASV, SmartCare). Best Pract Res Clin Anaesthesiol 2009;23(1):81-93

18 Liu N, Chazot T, Hamada S, et al. Closed-loop coadministration of propofol and remifentanil guided by bispectral index: a randomized multicenter study. Anesth Analg 2011;112(3):546-557

19 Liu N, Chazot T, Genty A, et al. Titration of propofol for anesthetic induction and maintenance guided by the bispectral index: closed-loop versus manual control: a prospective, randomized, multicenter study. Anesthesiology 2006;104(4):686-695

20 Puri GD, Kumar B, Aveek J. Closed-loop anaesthesia delivery system (CLADS) using bispectral index: a performance assessment study. Anaesth Intensive Care 2007;35(3):357-362

21 Janda M, Simanski O, Bajorat J, Pohl B, Noeldge-Schomburg GF, Hofmockel R. Clinical evaluation of a simultaneous closed-loop anaesthesia control system for depth of anaesthesia and neuromuscular blockade. Anaesthesia 2011;66(12):1112-1120

22 Eleveld DJ, Proost JH, Wierda JMKH. Evaluation of a closed-loop muscle relaxation control system. Anesth Analg 2005;101(3):758-764

23 Cavalcanti AB, Silva E, Pereira AJ, et al. A randomized controlled trial comparing a computer-assisted insulin infusion protocol with a strict and a conventional protocol for glucose control in critically ill patients. J Crit Care 2009;24(3):371-378

24 Rinehart J, Lee C, Cannesson M, Dumont G. Closed-loop fluid resuscitation: robustness against weight and cardiac contractility variations. Anesth Analg 2013;117(5):1110-1118

25 Kramer GC, Kinsky MP, Prough DS, et al. Closed-loop control of fluid therapy for treatment of hypovolemia. J Trauma 2008;64(4, Suppl):S333-S341

26 Ghosh S, Feng M, Nguyen H, Li J. Hypotension risk prediction via sequential contrast patterns of ICU blood pressure. IEEE J Biomed Health Inform 2016;20(5):1416-1426

27 Hatib F, Jian Z, Buddi S, et al. Machine-learning algorithm to predict hypotension based on high-fidelity arterial pressure waveform analysis. Anesthesiology 2018;129(4):663-674

28 Kendale S, Kulkarni P, Rosenberg AD, Wang J. Supervised machine-learning predictive analytics for prediction of postinduction hypotension. Anesthesiology 2018;129(4):675-688

29 Donald R, Howells T, Piper I, et al; BrainIT Group. Forewarning of hypotensive events using a Bayesian artificial neural network in neurocritical care. J Clin Monit Comput 2019;33(1):39-51

30 Celi LA, Hinske LC, Alterovitz G, Szolovits P. An artificial intelligence tool to predict fluid requirement in the intensive care unit: a proof-of-concept study. Crit Care 2008;12(6):R151

31 Nemati S, Holder A, Razmi F, Stanley MD, Clifford GD, Buchman TG. An interpretable machine learning model for accurate prediction of sepsis in the ICU. Crit Care Med 2018;46(4):547-553

32 Oh J, Makar M, Fusco C, et al. A generalizable, data-driven approach to predict daily risk of Clostridium difficile infection at two large academic health centers. Infect Control Hosp Epidemiol 2018;39(4):425-433

33 Parreco J, Hidalgo A, Parks JJ, Kozol R, Rattan R. Using artificial intelligence to predict prolonged mechanical ventilation and tracheostomy placement. J Surg Res 2018;228:179-187 
34 Kuo HJ, Chiu HW, Lee CN, Chen TT, Chang CC, Bien MY. Improvement in the prediction of ventilator weaning outcomes by an artificial neural network in a medical ICU. Respir Care 2015;60(11):1560-1569

35 Struck AF, Ustun B, Ruiz AR, et al. Association of an electroencephalography-based risk score with seizure probability in hospitalized patients. JAMA Neurol 2017;74(12):1419-1424

36 Beaulieu-Jones BK, Orzechowski P, Moore JH. Mapping patient trajectories using longitudinal extraction and deep learning in the MIMIC-III critical care database. Pac Symp Biocomput 2018;23:123-132

37 Parreco J, Hidalgo A, Kozol R, Namias N, Rattan R. Predicting mortality in the surgical intensive care unit using artificial intelligence and natural language processing of physician documentation. Am Surg 2018;84(7):1190-1194

38 Desautels T, Das R, Calvert J, et al. Prediction of early unplanned intensive care unit readmission in a UK tertiary care hospital: a cross-sectional machine learning approach. BMJ Open 2017;7(9):e017199

39 Weissman GE, Hubbard RA, Ungar LH, et al. Inclusion of unstructured clinical text improves early prediction of death or prolonged ICU stay. Crit Care Med 2018;46(7):1125-1132

40 Awad A, Bader-El-Den M, McNicholas J, Briggs J. Early hospital mortality prediction of intensive care unit patients using an ensemble learning approach. Int J Med Inform 2017;108:185-195

41 Delahanty RJ, Kaufman D, Jones SS. Development and evaluation of an automated machine learning algorithm for in-hospital mortality risk adjustment among critical care patients. Crit Care Med 2018;46(6):e481-e488

42 Rughani AI, Dumont TM, Lu Z, et al. Use of an artificial neural network to predict head injury outcome. J Neurosurg 2010;113(3):585-590

43 Raj R, Luostarinen T, Pursiainen E, et al. Machine learning-based dynamic mortality prediction after traumatic brain injury. Sci Rep 2019;9(1):17672

44 Dumont TM, Rughani AI, Tranmer BI. Prediction of symptomatic cerebral vasospasm after aneurysmal subarachnoid hemorrhage with an artificial neural network: feasibility and comparison with logistic regression models. World Neurosurg 2011;75(1):57-63, discussion 25-28

45 Paliwal N, Jaiswal P, Tutino VM, et al. Outcome prediction of intracranial aneurysm treatment by flow diverters using machine learning. Neurosurg Focus 2018;45(5):E7

46 Hollon TC, Parikh A, Pandian B, et al. A machine learning approach to predict early outcomes after pituitary adenoma surgery. Neurosurg Focus 2018;45(5):E8

47 Benjamin EJ, Blaha MJ, Chiuve SE, et al; American Heart Association Statistics Committee and Stroke Statistics Subcommittee. Heart disease and stroke statistics-2017 update: a report from the American Heart Association. Circulation 2017;135(10):e146-e603

48 Park E, Chang HJ, Nam HS. A bayesian network model for predicting post-stroke outcomes with available risk factors. Front Neurol 2018;9:699

49 Gupta S, Sharma DK, Gupta MK. Artificial intelligence in diagnosis and management of ischemic stroke. Biomed J Sci \& Tech Res 2019;13(3):9964-9967

50 Cuingnet R, Rosso C, Lehericy S, et al. Spatially regularized SVM for the detection of brain areas associated with stroke outcome. Paper presented at: Medical image computing and computer-assisted intervention MICCAI 13th International Conference 2010; Beijing, China;13:316-23

51 Bouts MJ, Tiebosch IA, van der Toorn A, Viergever MA, Wu O, Dijkhuizen RM. Early identification of potentially salvageable tissue with MRI-based predictive algorithms after experimental ischemic stroke. J Cereb Blood Flow Metab 2013;33(7):1075-1082

52 Asadi H, Dowling R, Yan B, Mitchell P. Machine learning for outcome prediction of acute ischemic stroke post intra-arterial therapy. PLoS One 2014;9(2):e88225

53 Asadi H, Dowling R, Yan B, Mitchell P. Intra-arterial therapy for basilar artery thrombosis: the role of machine learning in outcome prediction. PLoS One 2014;9:e88225

54 Asadi H, Dowling R, Yan B, Mitchell P. Is prognostication possible in patients with aneurysmal subarachnoid haemorrhage post endovascular treatment? Transl Biomed 2016;7(1):45

55 Madani A, Arnaout R, Mofrad M, Arnaout R. Fast and accurate view classification of echocardiograms using deep learning. NPJ Digit Med 2018;1:6

56 Shin H-C, RobertsK, LuL, et al. Learning to read chest x-rays: recurrent neural cascade model for automated image annotation. Paper presented at: 2016 IEEE Conference on Computer Vision and Pattern Recognition (CVPR); Las Vegas, NV; 2016;2497-2506

57 Sheng C, Li L, Pei W. Automatic detection of supporting device positioning in intensive care unit radiography. Int J Med Robot 2009;5(3):332-340

58 Gholami B, Phan TS, Haddad WM, et al. Replicating human expertise of mechanical ventilation waveform analysis in detecting patient-ventilator cycling asynchrony using machine learning. Comput Biol Med 2018;97:137-144

59 Papadelis C, Ashkezari SFS, Doshi C, et al. Real-time multi-channel monitoring of burst-suppression using neural network technology during pediatric status epilepticus treatment. Clin Neurophysiol 2016;127(8):2820-2831

60 Rajpurkar P, Hannun A, Haghpanahi M, et al. Cardiologist-level arrhythmia detection with convolutional neural networks Stanford Machine Learning Group and iRhythm Technologies, San Francisco, CA: ML Group; 2017

61 Clifford GD, Silva I, Moody B, et al. False alarm reduction in critical care. Physiol Meas 2016;37(8):E5-E23

62 Wu M, Ghassemi M, Feng M, Celi LA, Szolovits P, Doshi-Velez F. Understanding vasopressor intervention and weaning: risk prediction in a public heterogeneous clinical time series database. J Am Med Inform Assoc 2017;24(3):488-495

63 Dejournett L, Dejournett J. In silico testing of an artificial-intelligence-based artificial pancreas designed for use in the intensive care unit setting. J Diabetes Sci Technol 2016;10(6):1360-1371

64 Levin S, Toerper M, Hamrock E, et al. Machine-learning-based electronic triage more accurately differentiates patients with respect to clinical outcomes compared with the emergency severity index. Ann Emerg Med 2018;71(5):565-574.e2

65 Stonko DP, Dennis BM, Betzold RD, Peetz AB, Gunter OL, Guillamondegui OD. Artificial intelligence can predict daily trauma volume and average acuity. J Trauma Acute Care Surg 2018;85(2):393-397

66 Vranas KC, Jopling JK, Sweeney TE, et al. Identifying distinct subgroups of ICU patients: a machine learning approach. Crit Care Med 2017;45(10):1607-1615 